\title{
Randomness vs. fuzziness in managerial decision-making
}

\author{
Simona Hašková ${ }^{1, *}$ \\ ${ }^{1}$ The Institute of Technology and Business in České Budějovice, School of Expertness and Valuation, \\ Okružní 517/10, 37001 České Budějovice, Czech Republic
}

\begin{abstract}
Managers often deal with uncertainty of a different nature in their decision processes. They can encounter uncertainty in terms of randomness or fuzziness (i.e., mist, obscurity, inaccuracy or vagueness). In the first case (randomness), it can be described, for example, by probability distribution, in the second case (fuzziness) it cannot be characterized in such a way. The methodological part of the paper presents basic tools for dealing with the uncertainty of both of these types, which are techniques of probability theory and fuzzy approach technique. The original contribution of the theoretical part is the interpretation of these different techniques based on the existence of fundamental analogies between them. These techniques are then applied to the problem of the project valuation with its „internal“" value. In the first case, the solution is the point value of the statistical $\mathrm{E}[\mathrm{PV}]$, in the second case the triangular fuzzy number of the subjective E[PV]. The comparison of the results of both techniques shows that the fuzzy approach extends the standard outcome of a series useful information. This informative "superstructure" of the fuzzy approach compared to the standard solution is another original benefit of the paper.
\end{abstract}

Key words: uncertainty, probability, fuzziness, expected value, fuzzy number

\section{Introduction}

In the real world, much of the decision-making takes place in an environment where the consequences of the decisions cannot be predicted with certainty. This is due to the existence of internal and external factors that the decision-maker is able to influence only partially or not at all. In practice, however, it is in many cases quietly assumed that uncertainty in the sense of fuzziness (i.e. in the sense of mist, obscurity, inaccuracy or vagueness), whatever its nature, can be considered as randomness, which is a doubtful assumption.

We can only talk about randomness in conjunction with the elements of the universe whose objectively identified basic statistical characteristics are known. If nothing is known about it, it is uncertainty (see [1]). One of the reasons why it is necessary to distinguish between randomness and uncertainty is that they cannot be treated equally. Uncertainty is the main source of vagueness in many decision-making processes. We perceive it as a type of uncertainty that is associated with the use of intuitive (or only generally or linguistically

\footnotetext{
*Corresponding author: haskovas@post.cz
} 
defined) terms (for details see [2]). Mathematics models these concepts by means of fuzzy sets, i.e. by the classes in which there is no sharp transition between membership and nonmembership (this occurs when the characteristic function of the class is more than twovalued).

Decision-making in a fuzzy environment means a decision-making process in which goals and / or constraints, but not necessarily the whole system, are of fuzzy nature. This means that targets and / or limitations are classes that are not strictly defined. An example of a fuzzy constraint is: „The price of product A should be considerably higher than $\beta^{\text {“, }}$ where $\beta$ is a specified constant. Similarly, an example of a fuzzy target: ,y is located near $\mathrm{y}_{0}$ ", where $\mathrm{y}_{0}$ is a constant. The underlined words in these examples are the source of vagueness (see [3]). Fuzzy targets and fuzzy constraints can be defined as fuzzy sets in space of alternatives. Fuzzy decisions can be understood as intersection of given goals and constraints. The optimal decision is the set of points in the space of alternatives where the membership function of fuzzy decision achieves the best possible value.

In the case of randomness, the uncertainty concerns the membership or non-membership of the object in a non-fuzzy set. An example of randomness is the statement „The

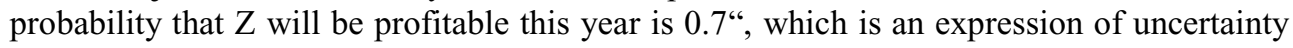
about Z's membership in a non-fuzzy set of profitable companies.

The difference between randomness and uncertainty is reflected in various mathematical techniques. In the first case, the techniques of probability theory help to cope with the existence of randomness; in the case of fuzziness, the technique of fuzzy approach is applied.

Fuzzy techniques are simpler than probability techniques in many respects because the concept of probability in probability theory corresponds to a simpler concept of membership function in fuzzy approach. In addition, for operations $a+b$, or respectively $a$. $\mathrm{b}$, where $\mathrm{a}, \mathrm{b}$ are the real numbers, the simpler operations $\max \{\mathrm{a}, \mathrm{b}\}$ and $\min \{\mathrm{a}, \mathrm{b}\}$ apply (more in [4]).

The aim of the paper is to put into context fuzzy approach and probabilistic approach, i.e. to specify the main principles and differences of both the techniques and to identify analogies between them. This is in the theoretical level the subject of the methodological part. The application part compares both approaches within the problem of quantifying the „,internal“ value of the reality close project of a developer company. The results of the approaches within the mini-study are discussed and interpreted. The conclusion summarizes the essentials, including the introduction of the original contributions of the paper.

\section{Methodological approach to random and vague phenomena in management}

Statistical procedures of probability theory in investment decision making are based on calculations of the expected values of future flows generated by the investment. The most commonly used criterion is the expected current value $\mathrm{E}[P V]$, which is the present value of the expected annual cash flows $\mathrm{E}\left[C F_{i}\right]$ in years $i=1,2$ to $n$ - see relation (1).

$$
\mathrm{E}[P V]=\sum_{i=1}^{n} \mathrm{E}\left[C F_{i}\right] / \prod_{j=1}^{i}\left(1+r_{j}\right)
$$

where $C F_{i}, i>0$ are net cash flows (positive or negative) generated by the project in $i$ - year of operation, $r_{j}$ is the positive discount rate per annum valid in the $j$-year of the project life, $\mathrm{E}$ is the symbol of the weighted average.

Probabilistic analysis of the cash flow can be used if the probabilities of possible outcomes are known, i.e. if the distribution of the frequency of possible outcomes is known. If it is not known, as it is typical in the case of demand for new products, most decision- 
makers rely on the knowledge and opinions of experts in predicting the time cash flow of the investment at the stage of its assessment.

In the literature, we find a number of authors who have dealt with the approach of evaluating investments in terms of the expected present value and its alternatives and the variance of the present value of probabilistic cash flows in different time ranges. Let us note, for example, Zinn et al. [5] who processed the formulas for the expected net present value, variance and semi-variance of net present values for different cash flow profiles at random time. In [6] Tufekci and Young presented the moments of net present value of probabilistic investment alternatives. The publication [7] compares two discount methods for the evaluation of multi-time stochastic income flows, which are identical and time independent.

The fuzzy method is based on the theory of fuzzy sets and represents an alternative approach in the case of uncertain variables characterized by the absence of any statistical description in the structure of the $C F_{i}$ values and / or uncertain values of the discount rates $r_{j}$.

The theory of fuzzy sets has its foundations in various versions of fuzzy logic that were created by adapting the binary numerical characteristics of propositional operators to the interval $\langle 0,1\rangle$. For example, if $|\mathrm{A}| \in\{0,1\}$, or respectively $|\mathrm{B}| \in\{0,1\}$ is the truth value of $\mathrm{A}$, or respectively $\mathrm{B}$, we can classify the classical truth tables for negation, conjunction, disjunction, and implication by the numerical characteristics of these operators: $|\neg A|=1-$ $|\mathrm{A}|,|\mathrm{A} \wedge \mathrm{B}|=\min \{|\mathrm{A}|,|\mathrm{B}|\},|\mathrm{A} \vee \mathrm{B}|=\max \{|\mathrm{A}|,|\mathrm{B}|\}$ and $|\mathrm{A} \rightarrow \mathrm{B}|=1$ for $|\mathrm{A}| \leq|\mathrm{B}|,|\mathrm{A} \rightarrow \mathrm{B}|=1-$ $(|A|-|B|)$, otherwise. Moving from the set $\{0,1\}$ to the interval $\langle 0,1\rangle$, we get the semantic form of Lukasiewicz's fuzzy propositional calculus, originally formulated by Lukasiewicz and Tarski ([8]). Fuzzy logic serves here only as a tool for the exact handling of fuzzy sets, the theory of which was published by L. A. Zadeh in 1965 - see [9].

In brief, the fuzzy set is a class of arranged pairs in which the first member is an element of the given universe of consideration, the second number comes from the interval $\langle 0,1\rangle$, which assigns it a degree of membership to a subset of the universe (i.e. to the support of the fuzzy set). This membership degree corresponds to the extent to which the element is compatible with the fuzzy set support. More specifically:

Let the set $U$ be a field of reasoning or discussion (universe), let $\mu_{\underline{A}}: U \rightarrow\langle 0,1\rangle$ be a membership function and let $\underline{A}=\left\{\left(y, \mu_{\underline{A}}(y)\right): y \in U\right\}$ be the set of all pairs $\left(y, \mu_{\underline{A}}(y)\right)$, in which the numbers $0 \leq \mu_{\underline{A}}(y) \leq 1$ state to the given $y \in U$ the degree of membership of the pair $\left(y, \mu_{\underline{A}}(y)\right)$ to the set $\underline{A}$. Then $\underline{A}$ is a fuzzy subset on the universe $U$. The significant characteristic of the fuzzy subset $\underline{A}$ is its support $U_{\underline{A}}=\left\{y: 0<\mu_{\underline{A}}(y) \leq 1, y \in U\right\} \subset U$. In terms of fuzzy logic is $\mu_{\underline{A}}(y)=\left|y \in U_{\underline{A}}\right|$, where $\left|y \in U_{\underline{A}}\right|$ denotes the degree of veracity of the proposition that $y$ is the element of the support of the fuzzy set $\underline{A}$. The element $y \in U$ with $\mu_{\underline{A}}(y)=0.5$ is called the crossover point in $\underline{A}$. At values greater than 0.5 , the element $y$ rather belongs to $U_{\underline{A}}$, at the values smaller it rather does not.

The fuzzy subset $\underline{A}$, whose support $U_{\underline{A}} \subset U \subset R$, where $R$ is the set of real numbers, and its function $\mu_{\underline{A}}$ is gifted by the property of normality and convexity, i.e. at least in the case of one element $x \in U_{\underline{A}}$, it applies $\mu_{\underline{A}}(x)=1$, and $\mu_{\underline{A}}\left(x^{\prime}\right) \geq \min \left\{\mu_{\underline{A}}\left(x_{1}\right), \mu_{\underline{A}}\left(x_{2}\right)\right\}$ for all $x^{\prime} \in\left\langle x_{1}\right.$, $\left.x_{2}\right\rangle \subset U_{\underline{A}}$, we call the fuzzy number. Theoretically, there may be different shapes of membership function $\mu_{\underline{A}}$ of fuzzy numbers: triangular, trapezoidal, bell, sinusoidal, cosinusoidal (see e.g. [10]). In such a way defined fuzzy numbers can be used to the formal representation of uncertain variables.

There is a significant analogy between the function $f(x)$, which is the probability density of a random variable $x$ and the function $\mu_{\underline{A}}(x)$, which is the degree of the membership of the element $x$ to the support of the uncertain variable (a fuzzy number $\underline{A}$ ). For example, a similar role that in the case of the random variable $x$ plays an average value or expected 
value $\mathrm{E}[x]$, which is the horizontal coordinate of the centre of gravity of the area under the function $f(x)$ in its definition field, in the case of the uncertain variable this role is played by the horizontal coordinate of the centre of gravity under the course of the function $\mu_{\underline{A}}(x)$ above the interval defined by fuzzy support $\underline{A}$.

This analogy can be used to solve a number of problems with variables that are out of any statistical description. A trusted spot estimate, which can hardly be obtained in such a case, can be replaced by the corresponding coordinate of the position of the centre of gravity of an adequate fuzzy number with a support matching to the set of all possible results. In practice, we often use this method to measure something that is difficult to quantify (such as the growth of quality of life) with something relatively close to what is easily measurable (e.g. GDP growth - see [11]). Let $\underline{A}=\left(\mathrm{A}_{\mathrm{L}}, \mathrm{A}, \mathrm{A}_{\mathrm{R}}\right)$ and $\underline{B}=\left(\mathrm{B}_{\mathrm{L}}, \mathrm{B}, \mathrm{B}_{\mathrm{R}}\right)$ be triangular fuzzy numbers in which the indices $L$ and $R$ mark the left and right edges of their supports. Let the middle numbers without an index be their subjectively expected values in which $\mu_{\underline{A}}(\mathrm{~A})=\mu_{\underline{B}}(\mathrm{~B})=1$ can be reasonably assumed (subjectively expected values are usually at the centre of the fuzzy number supports and in the case of the symmetrical probability densities they coincide with the statistically expected values ).

Through the algebraic operations of the $(+),(-),(+)$ and $(/)$ of the calculus of triangular fuzzy numbers developed in [12], from which let us mention $\underline{A}(+) \underline{B}=\left(\mathrm{A}_{\mathrm{L}}+\mathrm{B}_{\mathrm{L}}, \mathrm{A}+\mathrm{B}\right.$, $\left.\mathrm{A}_{\mathrm{R}}+\mathrm{B}_{\mathrm{R}}\right), \underline{A}(-) \underline{B}=\left(\mathrm{A}_{\mathrm{L}}-\mathrm{B}_{\mathrm{R}}, \mathrm{A}-\mathrm{B}, \mathrm{A}_{\mathrm{R}}-\mathrm{B}_{\mathrm{L}}\right), \mathrm{k}(+) \underline{A}=\left(\mathrm{k}+\mathrm{A}_{\mathrm{L}}, \mathrm{k}+\mathrm{A}, \mathrm{k}+\mathrm{A}_{\mathrm{R}}\right)$ and $\underline{A}(/) \underline{B}=\left(\mathrm{A}_{\mathrm{L}} / \mathrm{B}_{\mathrm{R}}\right.$, $\left.\mathrm{A} / \mathrm{B}, \mathrm{A}_{\mathrm{R}} / \mathrm{B}_{\mathrm{L}}\right)$, it is possible to formulate the fuzzy number $\underline{P V}=\left(\mathrm{PV}_{\mathrm{L}}, \mathrm{PV}, \mathrm{PV}_{\mathrm{R}}\right)$ in the case of uncertain cash flows (the fuzzy numbers $\underline{C F_{i}}$ ) and uncertain discount rates (the fuzzy numbers $\left.\underline{r}_{j}\right)$ as follows ([13]):

$$
\begin{gathered}
\mathrm{PV}_{\mathrm{L}}=\sum_{\mathrm{i}=1}^{\mathrm{n}}\left[\max \left\{\mathrm{CF}_{\mathrm{iL}}, 0\right\} / \prod_{\mathrm{j}=1}^{\mathrm{i}}\left(1+\mathrm{r}_{\mathrm{jR}}\right)+\min \left\{\mathrm{CF}_{\mathrm{iL}}, 0\right\} / \prod_{j=1}^{\mathrm{i}}\left(1+\mathrm{r}_{\mathrm{jL}}\right)\right], \\
\mathrm{PV}=\sum_{\mathrm{i}=1}^{\mathrm{n}} \mathrm{CF}_{\mathrm{i}} / \prod_{j=1}^{\mathrm{i}}\left(1+\mathrm{r}_{\mathrm{j}}\right), \\
\mathrm{PV}=\sum_{\mathrm{i}=1}^{\mathrm{n}}\left[\max \left\{\mathrm{CF}_{\mathrm{iR}}, 0\right\} / \prod_{j=1}^{\mathrm{i}}\left(1+\mathrm{r}_{\mathrm{jL}}\right)+\min \left\{\mathrm{CF}_{\mathrm{iR}}, 0\right\} / \prod_{j=1}^{\mathrm{i}}\left(1+\mathrm{r}_{\mathrm{j} R}\right)\right] .
\end{gathered}
$$

\section{Application of probabilistic and fuzzy approach}

This probability and fuzzy approach will be applied to a reality close managerial decisionmaking problem. Basic input information and task data are:

- The developer company decides to invest in a construction project of a modern residential building in the outskirts of the capital.

- Construction completion including the inspection of apartments is planned within two years from the construction launch. If there is a delay in the plan for a year, the company chooses between the option to complete the project with an annual delay or to sell the project in the third year at an estimated price of EUR 23 millions. The possibility of construction completion according to schedule in time or with an annual delay is equally probable.

- In case of completion of the construction, the apartments will be sold to personal ownership in the next year. The amount of budgeted income from the sale of the apartments (net income is the difference between the sum of revenues of the apartment sales and operating costs, paid fixed costs associated with an investment and income tax) depends on the size of the demand for apartments. 
- In the case of the apartment sale in the third year strong demand is estimated with $70 \%$ probability, weak demand with $30 \%$ probability; in the case of the one-year later sale, strong demand is estimated with a probability of $60 \%$ and weak demand with $40 \%$ probability.

- The discount rate of the project $r$ is equal to the average project capital costs of 15 $\%$. As the company does not intend to change the structure of its long-term funding sources in the near future, it can be considered a constant.

- The prediction of net incomes $\mathrm{N}_{31}$ and $\mathrm{N}_{32}$, or respectively $\mathrm{N}_{41}$ and $\mathrm{N}_{42}$ from the sale of apartments in the third, or respectively in the fourth year of the project is captured in Tab. 1.

Table 1. Total budgeted net project income

\begin{tabular}{|c|c|}
\hline Demand in the third year & Total net income from sale in the third year $\mathbf{N}_{\mathbf{3 1}}$ and $\mathbf{N}_{\mathbf{3 2}}$ \\
\hline Strong 0.7 & Eur 40 mil. \\
\hline Weak 0.3 & Eur 25 mil. \\
\hline Demand in the fourth year & Total net income from sale in the fourth year $\mathbf{N}_{\mathbf{4 1}} \mathbf{a} \mathbf{N}_{\mathbf{4 2}}$ \\
\hline Strong 0.6 & Eur 38 mil. \\
\hline Weak 0.4 & Eur 23 mil. \\
\hline
\end{tabular}

Source: Own processing.

The task of the manager is to evaluate the project with its maximum ,internal“ value using the $\mathrm{E}[\mathrm{PV}]$ criterion and to determine the highest possible investment cost that is acceptable for a project with an adequate risk rate if it is not to be loss-making. The aim of the study is to assess whether the criterion in question is the mainstay of the manager's decision in this case.

\subsection{Model of sequential decision-making situation}

Data of Tab. 1 are captured in the leaves of the decision tree shown in Fig. 1. The ellipses show situational nodes characterizing the state of the project implementation after the second year of its lifetime and the estimated level of demand for apartments in the third and fourth year. Rectangles represent the decision nodes (the decision whether to invest in the project and the decision whether to sell it in the event of its delay or to finish it). 


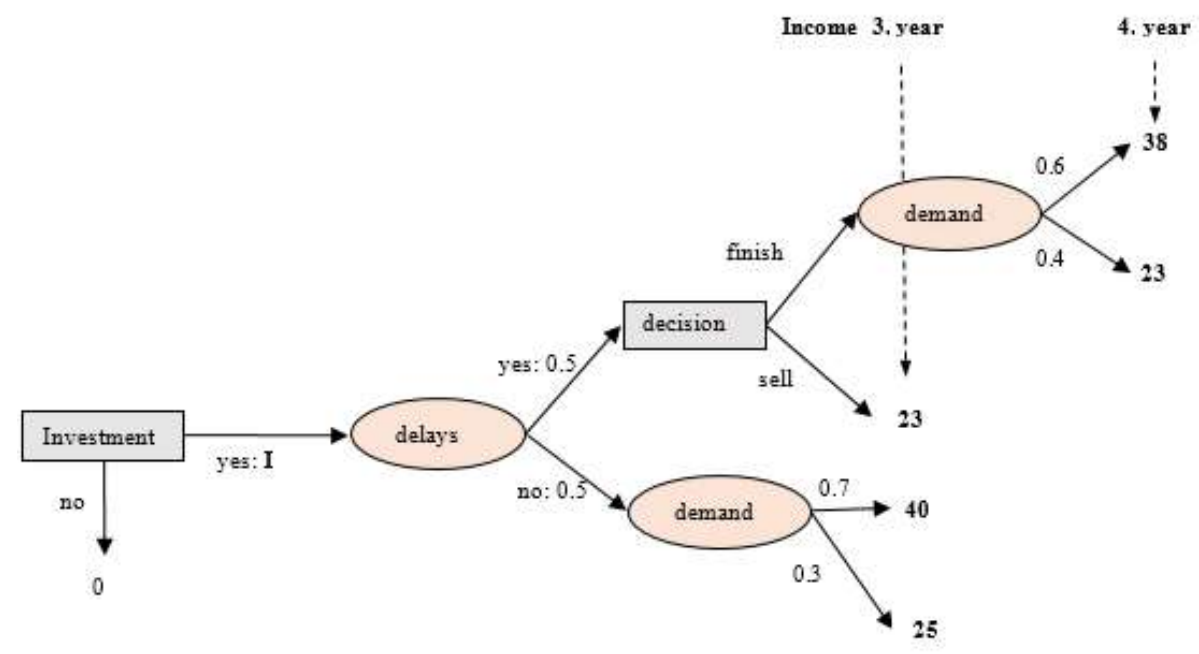

Fig. 1. Decision tree of the project of the construction of a modern residential building at current prices of $3 \mathrm{rd}$ and 4 th year in millions of Eur

Source: Own processing.

\subsubsection{Probabilistic evaluation based on data distribution and budgeted net income}

The model of the decision task in Fig. 1 is a sequential decision tree illustrating the statistical solution of the problem based on the unplanned forecasts of the point estimates of the random variables listed in Tab. 1. Construction completion time and demand level are also considered random variables described by an even distribution of probability density in the case of a delay, and in case of demand by some probability distribution according to the opinion of the financial manager of the company.

Given that there are generally positive numbers in the tree sheets with the exception of the root node, therefore $\mathrm{E}[\mathrm{PV}]>0$. The question of whether to invest asked in the root decision node can be reduced to the question of how much to invest $(I=$ ?). In the second decision node, in addition to the amount of EUR 23 million for the sale of the project, the amount calculated by the statistical approach $(0.6 \cdot 38+0.4 \cdot 23) / 1.15=27.8^{\dagger}$ is taken into account. This amount is greater than 23 - the decision is then known in advance and therefore this decision node can be omitted. The given step allows us to simplify the tree structure of Fig. 1 as shown in Fig. 2.

\footnotetext{
$\dagger$ The dot separating the numerical order refers to a number that is not of monetary significance, the comma separating the numerical order refers to the number, which is expressed in millions of Euros.
} 


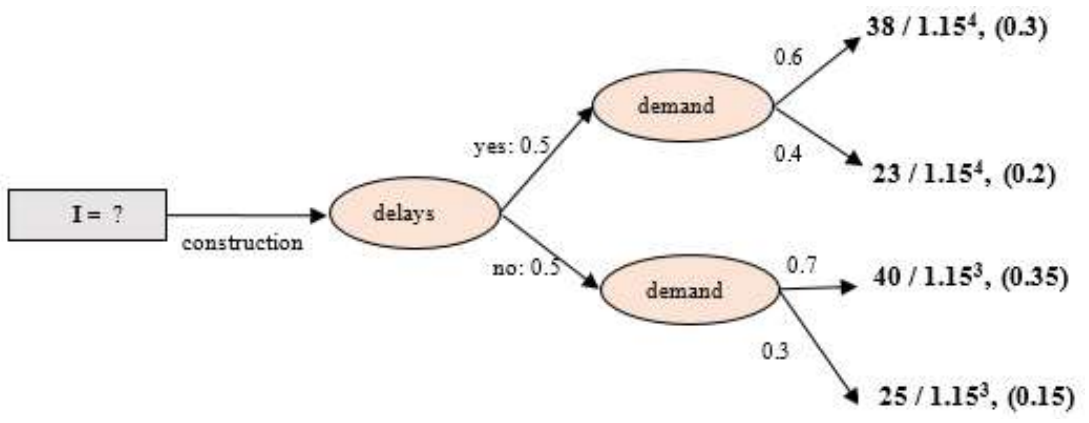

Fig. 2. The simplified tree of Fig. 1

Source: Own processing.

In it, four paths leading from the root to the sheets show four possible scenarios of the project run. Each of the scenarios is rated in its sheet by its current value $P V_{i j}=N_{i j} /(1+r)^{i}$ to the time $t=0$ of the project run (the tree root), where $\mathrm{N}_{\mathrm{ij}}$ are the net incomes from Tab. 1 . The brackets following the $\mathrm{PV}_{\mathrm{ij}}$ values are the estimated occurrence probabilities of the relevant scenario.

It applies: $\mathrm{E}[\mathrm{PV}]=0.3 \cdot \mathrm{PV}_{41}+0.2 \cdot \mathrm{PV}_{42}+0.35 \cdot \mathrm{PV}_{31}+0.15 \cdot \mathrm{PV}_{32}=0.5 \cdot(0.6 \cdot$ $\left.\mathrm{PV}_{41}+0.4 \cdot \mathrm{PV}_{42}\right)+0.5 \cdot\left(0.7 \cdot \mathrm{PV}_{31}+0.3 \cdot \mathrm{PV}_{32}\right)=0.5 \cdot\left(\mathrm{E}\left[\mathrm{PV}_{4}\right]+\mathrm{E}\left[\mathrm{PV}_{3}\right]\right)$.

The equality $\mathrm{E}[\mathrm{PV}]=0.5 \cdot\left(\mathrm{E}\left[\mathrm{PV}_{4}\right]+\mathrm{E}\left[\mathrm{PV}_{3}\right]\right)$ allows to reduce the original four scenarios to two scenarios and to redraw the tree from Fig. 2 to Fig. 3, showing that $E\left[\mathrm{PV}_{4}\right]$ $=32 / 1.15^{4}$ and $\mathrm{E}\left[\mathrm{PV}_{3}\right]=35,5 / 1.15^{3}$ with the same probability of occurrence of both of the scenarios.

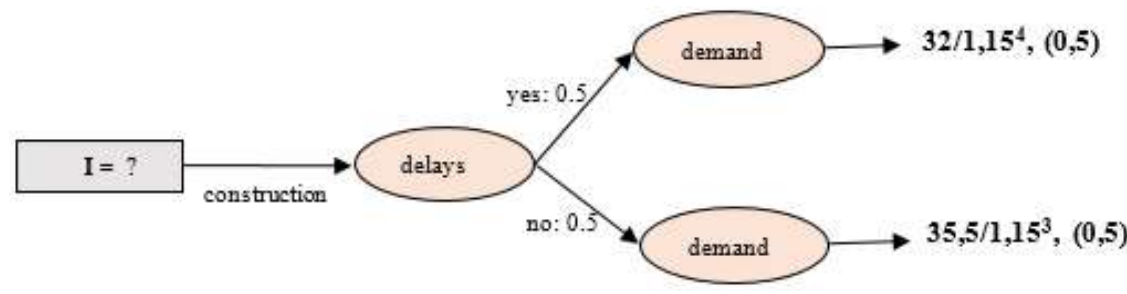

Fig. 3. Stochastic tree of the project after the reduction of four scenarios to two

Source: Own processing.

The solution sought is $\mathrm{E}[\mathrm{PV}]=0.5 \cdot\left(\mathrm{E}\left[\mathrm{PV}_{4}\right]+\mathrm{E}\left[\mathrm{PV}_{3}\right]\right)=0.5 \cdot\left(32 / 1.15^{4}+35,5 /\right.$ $\left.1.15^{3}\right)=20,82$ million EUR, which is simultaneously the ,internal“ value of the project. project. It also defines the highest possible investment cost for the lossless project.

\subsubsection{Evaluation of the project within fuzzy approach}

The basic method of considering data uncertainty in the fuzzy approach is the replacement of the estimated point values of the variables with triangular fuzzy numbers of type $(\mathrm{L} ; \mathrm{S}$; $\mathrm{P}$ ), where $\mathrm{L}$ is the smallest considered value, $\mathrm{P}$ is the largest, and $\mathrm{S}$ is the centre between them. This is an application of the general principle of indifference, which states that if there are more alternative results, and, at the same time, there is no relevant reason to prefer one or the other, it is appropriate to act as if all were equally probable. Then $\mathrm{S}$ is the subjectively expected value and (unlike the statistically expected value that is objective as being derived from the observation) it does not converge to any of the boundaries of the interval of possible values. In our model, the uncertain variable is the demand for apartments, from which the net income is also derived. In Fig. 1 the subjectively expected 
value of the fuzzy approach corresponds then to $30,5 / 1.15=26,5$, which is compared with the amount of EUR 23 million for the sale of the project (part 3.3.1, the second decision node). Similarly as in the case of the comparison of a statistically expected value, this subjective value is higher than 23 , therefore this decision node can be omitted and the model can be reduced in analogy to Fig. 3 on the fuzzy decision tree model in Fig. 4, wherein the second subjective value is 32,5 .

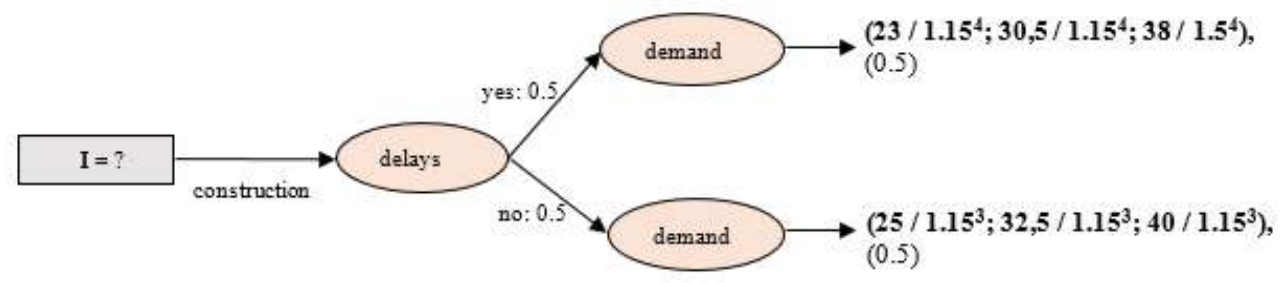

Fig. 4. Model of the considered project from a fuzzy approach perspective

Source: Own processing.

By the application of the tools of interval calculus mentioned in the methodological part, we obtain the fuzzy number of the sought solution in the form:

$\left(\mathrm{E}[\mathrm{PV}]_{\mathrm{L}}, \mathrm{E}^{*}[\mathrm{PV}], \mathrm{E}[\mathrm{PV}]_{\mathrm{R}}\right)=\left(0.5 \cdot 23 / 1.15^{4}+0.5 \cdot 25 / 1.15^{3} ; 0.5 \cdot 30,5 / 1.15^{4}+0.5 \cdot\right.$ $\left.32,5 / 1.15^{3} ; 0.5 \cdot 38 / 1.15^{4}+0.5 \cdot 40 / 1.15^{3}\right)=(\mathbf{1 4 , 8} ; \mathbf{1 9 , 4}$;

24),

in which the subjectively expected present value of potential net incomes from the sale of apartments is marked with the asterisk.

The fuzzy number $\left(14,8 ; 19,4^{*} ; 24\right)$ represents the interval of possible ,internal“ values of the project in which the left number can be understood as the value of the pessimistic scenario, the right number as the optimistic scenario and the middle number as the subjectively expected real value. At the same time, the interval of maximum investment costs is defined.

\subsection{Procedures analysis and results discussion}

Comparing the results of the standard and fuzzy analysis, we see that the fuzzy approach extends the standard result for a number of other useful information. Firstly, the „internal“ value of the $\mathrm{E}[\mathrm{PV}]$ project as a purely statistical variable is based on the weighted average of the relevant scenario values. In contrast, the ,internal“ fuzzy value of the project is a triangular fuzzy number $\underline{E[P V]}=\left(\mathrm{E}[\mathrm{PV}]_{\mathrm{L}}, \mathrm{E}^{*}[\mathrm{PV}], \mathrm{E}[\mathrm{PV}]_{\mathrm{R}}\right)$, whose extreme values define the lowest and highest possible present values with an average value as the subjectively expected value. The interval $\left(\mathrm{E}[\mathrm{PV}]_{\mathrm{L}}, \mathrm{E}[\mathrm{PV}]_{\mathrm{R}}\right)$ can be understood as the span between the result of the pessimistic and optimistic development of the project.

Furthermore, the fact that $E^{*}[\mathrm{PV}]=19,4$ is lower than $\mathrm{E}[\mathrm{PV}]=20,82$ only confirms the opinions of some authors e.g. in [14] that managers tend to overestimate their prognosis of positive outcomes - in the cash flow forecasts of projects the managers exaggerate positive flows and reduce negative flows. This trend corresponds to the probability distribution of the demand for apartments in the 3rd, or respectively 4th year. As a result the most of the large investment projects are completed late, they significantly exceed the budget and many of them will never meet the expectations of investors [15], [16]).

The manager, who derives from $\mathrm{E}[\mathrm{PV}]=20,82$ that the investment of $€ 20$ million in the project earns, for example, a minimal required gain of $€ 0,5$ million, is wrong. From the fuzzy analysis, it is clear that to reach this target, the initial investment should not exceed 
14,3 million Euro (i.e. for 0,5 less than $\mathrm{PV}_{\mathrm{L}}$ ); the question is whether the realization of the project would be feasible under these circumstances at all.

From the stated it can be seen that knowing the boundaries of the intervals of possible $\mathrm{E}[\mathrm{PV}]$, which the fuzzy approach provides, can be very significant for decision-makers.

\section{Conclusion}

In the world of management, most of the decision-making processes take place in an environment where the consequences of decisions made cannot be estimated with certainty. There is uncertainty of different nature that plays its role - it can be uncertainty in terms of randomness or uncertainty in the sense of fuzziness. The first mentioned can be expressed, for example, by probability distribution based on historically known data of the same or similar phenomena, in the latter case, it is not possible to describe it in such a way as the frequency of occurrence of the phenomenon is unknown. The theory of probability theory helps with the existence of uncertainty of the randomness type, in the case of fuzziness the technique of fuzzy approach can be successfully applied.

The most commonly used probability criterion in managerial decision making is the expected present value E[PV], which is the present value of expected annual cash flows $\mathrm{E}\left[C F_{i}\right]$ in years $i=1,2$ to $n$. In the fuzzy approach, the decision leans on the fuzzy number $\underline{E[P V]}=\left(\mathrm{E}[\mathrm{PV}]_{\mathrm{L}}, \mathrm{E}^{*}[\mathrm{PV}], \mathrm{E}[\mathrm{PV}]_{\mathrm{R}}\right)$ of uncertain cash flows (fuzzy numbers $\underline{C F_{i}}$ ) and uncertain discount rates (fuzzy numbers $\underline{r}_{j}$ ), where index L, or respectively R stands for left, respectively right border of the support of the respective fuzzy number.

The differences and analogies of approaches have been demonstrated to address the valuation of the project of the construction and sale of apartment units by its ,internal“ value. Comparing the results of probability and fuzzy analysis, it has emerged that the fuzzy approach extends the standard result for a number of useful information. In particular, $\mathrm{E}[\mathrm{PV}]$ is a weighted average whose calculation erases all boundary values of the project scenarios; the result is a number that carries less information than information of ,nonwiped out" scenarios. In contrast, the fuzzy approach generates limits of possible values of fuzzy number supports in which the middle value is a subjectively expected value not burdened by excessive optimism or scepticism of a manager regarding the occurrence of CF flows within individual scenarios. Knowledge of these limits is beneficial for investors with a different risk sensitivity relationship.

The described information advantages of fuzzy approach compared to the standard solution are the original benefits of the application part; the theoretical superstructure of the article is based on identifying the analogy between probabilistic approach and fuzzy approach.

\section{References}

1. R. E. Bellman, L. A. Zadeh, Decision-Making in a Fuzzy Environment. Management Science, 17(4), B-141, (1970)

2. J. M. Mendel, Uncertain rule-based fuzzy logic systems: introduction and new directions. Springer International Publishing, (2001)

3. L. A. Zadeh, The role of fuzzy logic in the management of uncertainty in expert systems. Fuzzy sets and systems, 11, 199-227, (1983)

4. L. A. Zadeh, J. Kacprzyk, Fuzzy logic for the management of uncertainty. WileyInterscience, (1992) 
5. C. D. Zinn, W. G. Lesso, B. Motazed, A probabilistic Approach to Risk Analysis in Capital Investment Projects. The Engineering Economist, 22(4), 239-260, (1977)

6. S.Tufekci, D. B. Young, Moments of the Present Worths of General Probabilistic Cash Flows Under Random Timing. The Engineering Economist, 32(4), 303, (1987)

7. V. Benzion, J. Yagil, On the Discounting Formula for a Stream of Independent Risky Cash Flows. The Engineering Economist, 32(4), 337, (1987)

8. T. J. Ross, Fuzzy logic with engineering application. Wiley, (2009)

9. L. A. Zadeh, Fuzzy Sets, Fuzzy Logic, And Fuzzy Systems. World Scientific Pub Co Inc, (1996)

10. C. Kahraman, Fuzzy multi-criteria decision making: theory and applications with recent developments. Springer US, (2008)

11. R. L. Ackoff, From data to wisdom. Journal of applied systems analysis, 16(1), 3-9, (1989)

12. C. Kahraman, Fuzzy versus probabilistic benefitúcost ratio analysis for public work projects. International Journal Applied Mathematics and Computer Science, 11(3), 705-718, (2001)

13. S. Haskova, Contribution to Economic Efficiency Evaluation of Projects in Terms of Uncertainty. $35^{\text {th }}$ International Conference on Mathematical Methods in Economics 2017 (MME 2016), 213-218, (2017)

14. D. Kahneman, D. Lovallo, Timid Choices and Bold Forecats: A cognitive perspective on Risk Taking. Management Science, 39(1), 17-31, (1993)

15. B. Flyvbjerg, Curbing Optimism Bias and Strategic Misrepresentation in Planning: Refernce Class Forecasting in Practice. European Planning Studies, 16(1), 3-21, (2008)

16. M. Vochozka, Using neural Networks to Determine the Financial Plan. Innovation Management, Entrepreneurship and Corporate Sustainability (IMECS 2016), 742-755, (2016) 